\title{
Summary report on synchronization, diagnostics and instrumentation
}

\author{
A.P. Freyberger ${ }^{a, *}$, G. Krafft ${ }^{\mathrm{a}, * *}$, \\ a Jefferson Lab, 12000 Jefferson Avenue, Newport News, VA. 23606
}

\begin{abstract}
The proceedings of Working Group 4 of the 2005 Energy Recovery Linac (ERL) workshop are summarized. Working Group 4 dealt with the challenging topic of beam diagnostics for ERL machines. Energy Recovery Linacs represent a challenge for beam diagnostics from several perspectives; invasive versus non-invasive diagnostics, longitudinal and transverse beam diagnostics, overall machine timing/synchronization and machine protection. Beam diagnostics for an ERL can benefit strongly from the experience at third generation light sources, recirculating linacs and presently operating ERLs. During the workshop there were presentations from all these communities, representing a large range operation experience in beam diagnostics. A brief summary Working Group 4 discussion is presented in this paper.
\end{abstract}

Key words: Energy Recovery Linac, Beam Diagnostics, PACS: 41.75.Ht, 41.85.Qg,

\section{Introduction}

This paper is the summary of the proceedings of Working Group 4 of the 2005 Energy Recovery Linac Workshop. The range of parameters for the different ERLs proposed or in operation is quite large and shown in Table 1.

The Working Group presentations were grouped according to beam properties and this summary continues that grouping. First the transverse beam diagnostics are summarized, followed by the longitudinal beam diagnostics. Within

\footnotetext{
* Corresponding author. Tel +1 757.269.6868

${ }^{* *}$ Corresponding author. Tel +1757.269 .7557$

Email address: freyberg@jlab.org, krafft@jlab.org (G. Krafft).
} 
Table 1

\begin{tabular}{|l|c|}
\hline Beam Property & Range \\
\hline \hline Current & $10^{-5}<I_{\text {beam }}<1 \mathrm{Amp}$ \\
\hline Emittance & $0.1<\epsilon<30 \mathrm{~mm}-\mathrm{mrad}$ \\
\hline Bunch Length & $0.1<T_{\text {bunch }}<100 \mathrm{psec}$ \\
\hline Energy Spread & $1<\sigma_{E}<1000 \mathrm{keV}$ \\
\hline Timing & $0.01<t<1 \mathrm{psec}$ \\
\hline Beam Loss & Fractional Loss $<10^{-6}$ \\
\hline Position Stability & $<\mu \mathrm{m}$ \\
\hline
\end{tabular}

Table summarizing the beam property ranges for existing and proposed ERLs

these two communities there is a strong effort on migrating from invasive techniques to non-invasive techniques that will allow for continuous monitoring of these properties. A summary of the machine timing and synchronization section follows. Improvements in timing and its distribution has implications for other diagnostics and systems notably the RF controls. The following two sections summarize the present status of beam position measurements and feedback and machine protection.

\section{Transverse Beam Profile}

Measurement of the transverse beam profile is usually done in an evasive manner. Evasive techniques include wire scanners, beam viewers, optical transition radiation monitors. These techniques will work for most ERL applications however they do not provide continuous monitoring and in most cases cannot perform the measurement with the beam at full power. The discussion focused on non-invasive techniques. A. Lumpkin summarized the many non-invasive techniques used at the Advanced Photon Source [1]. Of the several interesting developments he showed, the most exciting were the new results using diffractive transition radiation to measure the beam profile. The radiation is produced by the insertion of a metal edge near $[10 \sigma]$ the beam and the optical portion of the emitted radiation is easily observed on a CCD camera. Impedance budget and protection from high power beam strikes are a concern with such a monitor.

Synchrotron light in the optical portion of the spectrum is a convenient noninvasive beam monitor. In order to measure transverse beam sizes below the diffractive limit, interferometry is used. The synchrotron light interferometers at Jefferson Lab were presented by A. Freyberger [2]. These devices are based on those built by Mitsuhashi [3,?]. The transverse beam size is a related to the 
visibility of the interference pattern, and a minimum spot resolution of $5 \mu \mathrm{m}$ can be achieved with a very good CCD camera. The JLAB interferometers have been used very successfully to continuously monitor the energy spread during nuclear physics experiments and are fully integrating into the machine operation.

\section{Longitudinal Beam Profile}

During this workshop a substantial portion of the time was devoted towards discussing various methods of determining the longitudinal distribution of the bunch, or more generally the longitudinal phase space of the bunch. The techniques were largely taken from present thinking about such diagnostics from the X-ray laser community with one notable exception that is discussed below. In this section we summarize the ideas presented, for more information one should consult either the references given, or the conference proceedings of recent free electron laser conferences.

The electro-optic method of determining the beam time profile was discussed[12]. The present resolution of such measurements is around $300 \mathrm{fsec}$, depending to some extent on the beam energy. This technique can also be used to determine the arrival time to the level of 30 fsec. Its principal strengths are that the measurement is nondestructive and can measure single high charge bunches. For example at even $100 \mathrm{pC}$ and $50 \mathrm{MeV}$ electron beam energy, a 600 fsec FWHM bunch may be analyzed. Different crystals than are standard now may lead to improved performance in the future, and many labs are now involved in the development of electro-optic longitudinal profile diagnostics.

Longitudinal phase space tomography was discussed as a method to obtain a more complete longitudinal phase space distribution. In this method the phase space is projected on various different axes of the phase space and reconstructed using mathematical techniques already highly developed in the medical imaging field. The present resolution of the techniques is about 100 fsec in time and $3 \mathrm{keV}$ ( $100 \mathrm{MeV}$ ) in energy, depending in detail on the resolution of the energy spectrometer. A principal shortcoming of this technique is that it assumes, and hence requires, beam stability over the multiple shots used to make the various projections. Implementation made so far have been destructive to normal beam operation. A variant of this idea, used at Stanford to obtain detailed time profiles, is to adjust $R_{56}$ in a bunching region and the offset phase in an accelerating cavity so the total longitudinal transfer matrix to the point where an image is made is as in a transverse focal point. Under this condition the image is completely independent of any initial energy spread in the longitudinal phase distribution. 
Zero phasing profile measurements, either with a longitudinal cavity as performed at Jefferson Lab [9] and BNL [10], or with a deflecting cavity as performed at SLAC [11], are highly destructive but offer high resolution of order 10 fsec. If the deflecting cavity method is combined with energy analysis in the plane transverse to the deflection direction, a detailed phase space distribution may be obtained simply by optical analysis of beam images produced with either transition radiation or electroluminesent viewers.

An idea extensively discussed at the sessions was a way to make the destructive methods of beam analysis almost nondestructive. In an ERL with an RF CW electron beam it is possible to occasionally, that is with low duty factor, deflect a small portion of the beam off the beamline for detailed measurement. One operates under the assumption, probably good for a CW ERL, that the beam properties being measured in the deflected beam are not appreciably changed by the deflection method used and reflect the beam properties of the high duty factor undeflected portion of the electron beam in detail.

Another beam diagnostic method is to measure the beam longitudinal transfer function [7]. While not giving data that can easily be turned into a longitudinal bunch distribution, this device is quite useful for determining that the RF elements that are responsible for bunching are properly set. It also allows, given that a properly characterized bunching program has been achieved previously, a means to rapidly return machine conditions to it proper state. As such, because the measurements may be done and interpreted relatively rapidly, such devices are particularly useful during the commissioning and operations phase at an operating accelerator. As summarized in a paper at this conference [8], such phase transfer devices have evolved into the major longitudinal dynamics diagnostic at each of the Jefferson Lab recirculated linacs.

\section{Machining Timing and Synchronization}

There are two separate, but related, issues that were discussed about machine synchronization for ERLs [13]. The broader of the two issues regards distributing synchronized master reference signals over substantial (of order $1 \mathrm{~km}$ ) distances with timing errors at the level of 10 fsec. The narrower of the two issues, regards the accuracy with which one may then lock a local control system, for example the phase of an RF voltage in a cavity, to one of these stabilized reference systems. As a subset of the second class of issues one has locking laser pulses to master references at the level of tens of fsec, which is related to how precisely the beam pulses are locked to the rest of the RF systems in the extended linac.

In dealing with all of these issues much work has been accomplished as a 
result of work from the X-ray laser community, and it became clear during the conference that many of the solutions proposed there could be beneficially applied to ERLs. For example, if one needs a mode-locked laser as the source of electrons in an ERL beam, it makes sense to derive the laser pulses from a very high precision Optical Master Oscillator. The resolution of such clocks these days is about 50 fsec now, and should be of order 10 fsec in a few years as a result of development work for X-ray lasers presentation by. Present examples have low noise above $10 \mathrm{kHz}$ as free-running laser, and can be locked to a microwave, or other, oscillator for long term stability. Similarly, all-optical absolute clocks were discussed with 45 attosecond it rms stability when integrated from $\mathrm{mHz}$ to $10 \mathrm{MHz}$ presentation by. Possibly they can be developed with timing precision about 1000 times better than the best microwave oscillators, eliminating the need for a more traditional microwave master oscillator. If such devices as photocathode sources can be accomplished, then the lion's share of the rest of the synchronization problem reduces to the problem of distributing synchronization signals properly.

One of the nice features of the all-optical clock is that it supports absolute synchronization of signals at widely separated stations. For example, if the time difference between outgoing and reflected pulses on an optical fibre were continuously monitored by autocorrelation techniques and corrected to high precision, then the pulses arriving at the far end of the fibre can be absolutely synchronized as long as any time delay mechanism experienced by the pulses happen in traversing both directions on the fibre. Presumably a complete accelerator installation would consist of several stations absolutely synchronized, which act to distribute master signals over shorter lines to control, for example, linac RF phases.

Simrock, Ludwig, and Schlarb [13] have presented much of the most recent data on the performance of optical clocks and much greater detail then here on the ideas and the need to synchronize systems in the X-FELs. They have also given extensive information on the performance of locking of the RF phases in superconducting cavities to a given external microwave source. As a brief summary, the phase of an L-band cavity can be locked to the source such that the residual fluctuations are at the level of 0.01 degrees of RF phase using a control system that is essentially digital in nature. To summarize their main conclusions: (1) The present state-of-the art in synchronization systems is at the fsec level. At this level available commercial low noise phase and amplitude detectors contribute to jitter so a careful selection of components, low noise design, and temperature stabilizations and self-calibrating techniques are needed to achieve this level. (2) Such good performance in a realistic and noisy accelerator environment needs to be demonstrated. (3) In order to quantify, and minimize the jitter in, e. g., beam arrival time, one should develop and implement complete error budgets for all potential sources of arrival time jitter error. 


\section{Beam Position}

Measuring the centroid of the beam with stripline or button rf pick-ups is a very mature technology. Such beam position monitors (BPM) will work for an ERL as well. The improvement to BPM systems is in the new digital electronics using the new field programmable gate arrays (FPGA) to add functionality to the BPM. A talk on the digital BPM and feedback system at T. Schilcher presented some of the features of such a system installed at SLS [5]. This system has achieved sub-micron stability and a $4 \mathrm{kHz}$ sample and update rate.

Additionally, two talks on using high order modes (HOM) of the accelerating structures to measure the beam position were presented by Sawamura and Firsch [6]. This relationship between the HOM strength and beam position relative to the cavity center has two potential uses. It can be used to measurement the alignment of the accelerating structures and thereby provide better modeling information. Additionally if space along the beam is so restricted [i.e. in the injector region] that there is no room for traditional BPM structures, then this HOM technique might provide an alternative.

\section{Machine Protection}

Overview of machine protection concepts were presented by H. Schlorb and K. Jordan gave a presentation on the machine protection of the JLAB FEL. Both talks emphasized machine protection based on known concepts and technologies. An ERL would have the same concepts of different states or modes. Diagnostic mode or tune mode for establishing a good orbit through the machine before allowing CW beam delivery. Machine protection and beam loss monitors presently in use at other machines (i.e. photo-multiplier tubes and ion chambers) should work at ERL machines. It was pointed out that machine protection must include the experimental lines as well as the injector.

One of the features of an ERL is the large stored power of the beam. The peak stored power of a $100 \mathrm{~mA} 6 \mathrm{GeV}$ ERL is 600MWatts and a fractional loss of $1 \mathrm{ppm}$ represents $600 \mathrm{Watts}$ of power or 100nA of beam current. Experience at JLAB has shown that a continuous loss of at this level is sufficient to warm up a flange and open up a vacuum leak. Peter Cameron presented a proposal to perform a differential current measurement using two DC current transformer measurements on the ERL test facility at BNL which has a maximum beam energy of $40 \mathrm{MeV}$ [14]. The two transformers are electrically connected such that the current measured in the injection line nulls the signal measured in the dump line. The technique is limited by the noise and estimates suggest 
a 1 part in $10^{4}$ measurement of the loss. This is sufficient for the ERL test facility and hopefully this effort results in some insights on how to improve loss measurements by two orders of magnitude. This is an outstanding issue for high power ERLs.

\section{Summary}

In this working group many aspects of the electron beam diagnostics systems for new Energy Recovered Linacs were presented and reviewed. The work was divided into several broad categories: transverse diagnostics, for position, profile, and phase space; longitudinal diagnostics for time-of-arrival, longitudinal profile, and longitudinal phase space; the synchronization systems needed as a fundamental aspect of producing properly bunched beam bunches; and aspects of machine protection and beam halos, which will be especially important for high average current ERLs. The references to this paper provide a good starting point to explore the subject matter in more depth.

At some point, because a laser beam produces the electron beam in the first place and thus are so instrumental in establishing the initial beam properties, more extensive discussions on laser beam diagnostic requirements and capabilities should be subjects for discussions in future meetings.

\section{References}

[1] A. Lumpkin, "Nonintercepting Diagnostics for Transverse Beam Parameters: From Rings to ERLs", in these proceedings

[2] A. Freyberger, "Non-invasive Energy Spread Monitor for the JLAB Experimental Program via Synchrotron Light Interferometers", in these proceedings

[3] T. Mitsuhashi, "Beam Profile Measurement by SR Interferometers", in Proceedings of the Joint U.S.-CERN-Japan-Russia School on Particle Accelerators, Montreux, Switzerland, 1998.

[4] T. Mitsuhashi, "Faraday Cup Award Talk: 12 Years of SR Monitor Development at KEK", in Proceedings of the $11^{\text {th }}$ Beam Instrumentation Workshop, Knoxville, Tennessee USA 2004.

[5] T. Schilcher,

M.

Böge, B. Keil, P. Poller, and V. Schlott, "Commissioning and Operation of the SLS Fast Orbit Feedback" in Proceedings of EPAC 2004, Lucerne, Switzerland. http://accelconf . web.cern.ch/AccelConf/e04/PAPERS/THPLT024.PDF. 
[6] M. Sawamura and R. Nagai, "Beam Position Monitor with HOM couplers", in these proceedings.

[7] G. A. Krafft, AIP Conf. Proc. 367, 46 (1995).

[8] G. A. Krafft, "Phase Transfer Function Measurements at the Jefferson Lab Recirculated Linacs", in these proceedings.

[9] D. X. Wang, G. A. Krafft, and C. K. Sinclair, Phys. Rev. E, 57, 2283 (1998).

[10] W. S. Graves, et al., Proc. of the 2001 Part. Acc. Conf., 2860 (2001).

[11] A. Akre, et al., Proc. of the 2002 European Part. Acc. Conf., 1882 (2002).

[12] H. Loos, "Longitudinal phase space tomography and its implementation in energy recover linacs", in these proceedings.

[13] S. Simrock, F. Ludwig and H. Schlarb, "Synchronization Systems for ERLs", in these proceedings.

[14] P. Cameron, "Differential Current Measurement in the BNL Energy Recovery Linac Test Facility", in these proceedings. 